\title{
Análise e georreferenciamento da mortalidade materna em Aracaju de 2010 a 2018
}

\author{
Analysis and georeferencing of maternal mortality in Aracaju from 2010 to 2018 \\ Análisis y georreferenciación de la mortalidad materna en Aracaju de 2010 a 2018
}

\section{Resumo}

O óbito materno é definido como aquele que ocorre durante a gestação ou até 42 dias após o parto e possui relação direta ou indireta com esse processo. Devido a sua evitabilidade é considerada uma grave violação dos direitos humanos. Este estudo objetiva analisar os casos de mortalidade materna em Aracaju de 2010 a 2018, segundo suas causas, características sociodemográficas, obstétricas e distribuição geográfica. Trata-se de um estudo ecológico, de base territorial, do tipo série temporal, realizado em Aracaju/SE, tendo como amostra os dados obtidos através da Vigilância Epidemiológica Municipal e do DATASUS referente aos óbitos maternos que ocorreram no município de 2010 a 2018. Para análise estatística foi utilizado o software R e para o mapeamento dos óbitos o QGIS 3.8. A Razão de Mortalidade Materna manteve-se instável durante o período analisado. O ano de 2015 apresentou a menor taxa e 2011 a maior. O perfil sociodemográfico evidencia que a maioria era de cor Preta/Parda, solteira, com escolaridade de 8 a 11 anos de estudo e apenas donas de casa. A média de idade foi de 29,47 anos. Um número expressivo dos óbitos foi relacionado a causas obstétricas diretas. A maioria ocorreu no puerpério, em estabelecimentos públicos e foram classificados como provavelmente evitáveis. As zonas Norte e o Oeste concentraram maior ocorrência de casos. A mortalidade materna no município manteve-se acima da média preconizada para o país, com destaque para as causas obstétricas diretas e o predomínio da população preta/parda que chama a atenção para a vulnerabilidade desse grupo.

Palavras-chave: Mortalidade materna; Saúde pública; Mapeamento geográfico.

\begin{abstract}
Maternal death is defined as that which occurs during pregnancy or up to 42 days after delivery and has a direct or indirect relationship with this process. Due to its avoidability it is considered a serious violation of human rights. This study aims to analyze the cases of maternal mortality in Aracaju from 2010 to 2018, according to their causes, sociodemographic, obstetric characteristics and geographic distribution. This is an ecological study, territorially based, of the temporal series type, carried out in Aracaju/SE, using as a sample the data obtained through the Municipal Epidemiological Surveillance and DATASUS referring to maternal deaths that occurred in the municipality from 2010 to 2018. For statistical analysis, the Software R was used and QGIS 3.8 was used for the mapping of deaths. The Maternal Mortality Ratio remained unstable during the analyzed period. The year 2015 had the lowest rate and 2011 the highest. The sociodemographic profile shows that the majority were black/brown, single, with schooling from 8 to 11 years of schooling and only housewives. The mean age was 29.47 years. A significant number of deaths were related to direct obstetric causes. Most occurred in the puerperium, in public establishments and were classified as probably preventable. The North and West zones concentrated a higher occurrence of cases. Maternal mortality in the
\end{abstract}


municipality remained above the average recommended for the country, with emphasis on direct obstetric causes and the predominance of the black/brown population that draws attention to the vulnerability of this group.

Keywords: Maternal mortality; Public health; Geographic mapping.

\begin{abstract}
Resumen
La muerte materna se define como aquella que ocurre durante el embarazo o hasta 42 días después del parto y tiene una relación directa o indirecta con este proceso. Debido a su evitabilidad se considera una grave violación de los derechos humanos. Este estudio tiene como objetivo analizar los casos de mortalidad materna en Aracaju de 2010 a 2018 , según sus causas, características sociodemográficas, obstétricas y distribución geográfica. Se trata de un estudio ecológico, de base territorial, del tipo de serie temporal, realizado en Aracaju/SE, utilizando como muestra los datos obtenidos a través de la Vigilancia Epidemiológica Municipal y DATASUS referidos a muertes maternas ocurridas en el municipio de 2010 a 2018. Para el análisis estadístico se utilizó el Software R y QGIS 3.8 para el mapeo de muertes. La tasa de mortalidad materna se mantuvo inestable durante el período analizado. El año 2015 tuvo la tasa más baja y 2011 la más alta. El perfil sociodemográfico muestra que la mayoría eran negros/marrones, solteros, con escolaridad de 8 a 11 años de escolaridad y solo amas de casa. La edad media fue de 29,47 años. Un número significativo de muertes se relacionaron con causas obstétricas directas. La mayoría ocurrieron en el puerperio, en establecimientos públicos y fueron clasificados como probablemente prevenibles. Las zonas Norte y Oeste concentraron una mayor incidencia de casos. La mortalidad materna en el municipio se mantuvo por encima del promedio recomendado para el país, con énfasis en las causas obstétricas directas y el predominio de la población negra/morena que llama la atención sobre la vulnerabilidad de este grupo.
\end{abstract}

Palabras clave: Mortalidad materna; Salud pública; Mapeo geográfico.

\title{
1. Introdução
}

A Organização Mundial da Saúde (OMS) define a Mortalidade Materna (MM), como a morte da mulher que acontece durante a gestação, parto ou até 42 dias após o parto (World Health Organization, 2019). Cerca de 92\% dos óbitos maternos são considerados evitáveis, caracterizando-os como um grave problema de saúde pública e violação dos direitos humanos (Mascarenhas et al., 2017; Vanderlei \& Frias, 2015; Freitas-Júnior, 2020).

A Razão de Mortalidade Materna (RMM) é um indicador que subsidia avaliações populacionais e reflete baixos índices socioeconômicos, desigualdades de gênero e déficits assistenciais, haja visto que grandes partes desses óbitos são precoces e evitáveis (Barreto, 2021). Tais apontamentos contrapõem a premissa de uma assistência qualificada durante o ciclo gravídicopuerperal, sobretudo no contexto de sistemas públicos de saúde. Ademais, existe uma complexidade de fatores elementares interligados a MM, desafiando gestores e a sociedade, especialmente em países subdesenvolvidos e em desenvolvimento (Ruas et al., 2020).

Dessa forma, estratégias voltadas para mitigação dos índices de MM são historicamente desenvolvidas no âmbito internacional, a exemplo dos Objetivos de Desenvolvimento Sustentável, que sucedem os Objetivos de Desenvolvimento do Milênio, em que a redução dos óbitos maternos permanece como meta global pactuada para 70 mortes por 100 mil nascidos vivos (NV) até o ano de 2030 NV (Instituto de Pesquisa Econômica Aplicada, 2018). Contudo, a estimativa mais recente da OMS é que em 2017 tenham ocorridos 211 óbitos/100 mil NV (World Health Organization, 2019). Hodiernamente, estima-se que cerca de 830 mulheres morrem diariamente no mundo por complicações evitáveis da gestação (Vanderlei \& Frias, 2015 ; B. L. Barreto, 2021).

No Brasil destaca-se a Rede Cegonha e a Política Nacional de Atenção Integral à Saúde da Mulher, ambas vislumbram a garantia de direitos sexuais e reprodutivos das mulheres, atenção à saúde ampla, de qualidade e humanizada e a redução da MM. Outra iniciativa foi a criação dos Comitês de Mortalidade Materna os quais visam a identificação de fatores que determinem possíveis causas, subsidiando a tomada de medidas para prevenir a sua reincidência (Brasil, 2007; Brasil, 2011; Brasil \& Ministério da Saúde, 2009; Portaria N 1.119, de 5 de junho de 2008, 2008; Vanderlei \& Frias, 2015).

Apesar dos esforços, foram evidenciados 20.229 óbitos maternos no território brasileiro no período entre 2006 a 2017 , com ênfase para as regiões Norte e Nordeste cujas taxas de mortalidade foram de 71,9 e 74,7 óbitos por 100 mil Nascidos Vivos (NV) respectivamente, ultrapassando a taxa nacional de 62,3 óbitos por 100 mil NV no período estudado (Rodrigues et al., 2019). 
Dentre os estados do Nordeste, destacaram-se a Bahia (26,34\%), Maranhão (18,21\%), e Pernambuco (14,50\%). Sergipe apresentou o menor percentual de óbito (4,02\%) (Torres et al., 2021).

Diante do exposto, é imprescindível e relevante a análise dos óbitos maternos, visando compreender os fatores associados a tal agravo de saúde. Assim, esse estudo teve por objetivo analisar os casos de mortalidade materna em Aracaju no período de 2010 a 2018, segundo suas causas, características sociodemográficas e obstétricas, bem como a sua distribuição geográfica.

\section{Metodologia}

Trata-se de um estudo ecológico, do tipo série temporal, realizado no município de Aracaju, capital sergipana, que apresenta uma extensão territorial de 182,163 km² e uma população estimada para 2020 de 664.908 pessoas (Instituto Brasileiro de Geografia e Estatística, 2010).

Foram incluídos todos os casos de MM no município de Aracaju no período de 2010 a 2018, conforme conceito da OMS. Os dados referentes à MM e NV foram obtidos respectivamente das plataformas online do Sistema de Informação de Mortalidade (SIM) e Sistema de Informação de Nascidos Vivos (SINASC) do Ministério da Saúde (MS) - Departamento de Informática do SUS (DATASUS)/Sergipe, por meio da Vigilância Epidemiológica Municipal. Dados sociodemográficos e referentes ao município foram obtidos na plataforma de dados do Instituto Brasileiro de Geografia e Estatística (IBGE) (Instituto Brasileiro de Geografia e Estatística, 2021; Brasil, 2019).

Foi elegível como variável dependente o óbito materno e como variáveis independentes as características sociodemográficas, obstétricas e do serviço de saúde (faixa etária, cor/raça, escolaridade, estado civil e ocupação, período de ocorrência do óbito, momento do óbito, local de ocorrência do óbito, estabelecimento de ocorrência do parto/aborto, estabelecimento de ocorrência do óbito, realização do pré-natal, pré-natal adequado, parto de assistência adequada, evitabilidade do óbito, fatores de evitabilidade, classificação do óbito, óbito na declaração de óbito).

Para coleta de dados foi utilizado um instrumento estruturado abrangendo dados presentes na declaração de óbito (DO) e do comitê de investigação de óbitos (Borges, 2012). Os dados coletados foram submetidos à dupla digitação em uma planilha informatizada no programa Microsoft Office Excel®. Foi construído o indicador Razão de Mortalidade Materna (RMM), preconizada pelo MS, uma vez que seu numerador foi o número de óbitos maternos e seu denominador o número total de NV; o resultado por sua vez foi multiplicado por 100.000, seguindo padrão internacional (Portaria n ${ }^{\circ}$ 1.459, de 24 de junho de 2011, 2011; Carreno et al., 2014).

As variáveis qualitativas foram categorizadas e analisadas quanto às frequências e percentuais, já para as quantitativas foi preconizado a média e desvio padrão. Foi implementado análise bivariada para testar relação entre óbitos maternos e características sociodemográficas, obstétricas e serviço de atenção saúde. Variáveis qualitativas foram testadas pelo Quiquadrado (32) (Pearson, 1900) e quantitativas pelo teste de Mann-Whitney (Mann \& Whitney, 1947). Foi adotado nível de significância de 5\%. O software utilizado para a análise estatística foi o R, versão 3.6.1 (The R core team, 2018). Na análise espacial dos óbitos, foi utilizado o método de Kernel e o software QGIS 3.8 (QGIS Development Team, 2019).

O referido foi aprovado pelo Comitê de Ética em Pesquisa da Universidade Tiradentes - UNIT, com parecer $\mathrm{n}^{\circ}$ 3.286.413. Garantiu-se o cumprimento das normas da resolução 466/2012 do Conselho Nacional de Saúde.

\section{Resultados e discussão}

Foram identificados 49 óbitos maternos no período de 2010 a 2018 na capital do estado de Sergipe. O ano de 2015 apresentou a menor RMM do período (0/100 mil NV), seguido do ano de 2012 (21,33/100 mil NV). Em contrapartida, o ano de 2011 apresentou a maior taxa (123,17/100 mil NV), seguido de 2010 (96,08/100 mil NV) (Tabela 1). 
Quadro 1: Distribuição da RMM dos óbitos maternos em Aracaju de 2010 a 2018.

\begin{tabular}{|c|c|c|c|}
\hline Variável\Categorias & Óbitos & NV & RMM \\
\hline ANO DE ÓBITO & & & 96,08 \\
\hline 2010 & 9 & 9.367 & 123,17 \\
\hline 2011 & 12 & 9.742 & 21,33 \\
\hline 2012 & 2 & 9.375 & 53,33 \\
\hline 2013 & 5 & 9.374 & 62,59 \\
\hline 2014 & 5 & 9.586 & 0 \\
\hline 2015 & 0 & 9.662 & 56,38 \\
\hline 2016 & 5 & 8.867 & 77,19 \\
\hline 2017 & 7 & 9.068 & 42,73 \\
\hline 2018 & 4 & 9.359 & \\
\hline
\end{tabular}

Fonte: Vigilância Epidemiológica municipal (2019); Brasil (2019).

As taxas de RMM foram discrepantes no período do estudo. Mesmo diante desse fato percebeu-se que na maior parte dos anos essa taxa manteve-se elevada com uma média de aproximadamente 60 óbitos/100 mil NV, permanecendo ainda acima da meta preconizada pela OMS de 20 óbitos/ 100 mil NV (World Health Organization, 2019). A nível nacional foi observada uma redução de 8,4\% da RMM entre os anos de 2017 e 2018, em que o país passou de 64,5 para 59,1 óbitos/ 100 mil NV. No Nordeste essa redução foi de $8,3 \%$ no mesmo período, porém a região ainda é considerada de risco para a mortalidade materna (Guerra et al., 2016; Brasil et al., 2020).

Estudos realizados em estados da região Nordeste revelam tendência de aumento da RMM. A RMM entre 2007 a 2016 na Paraíba apresentou um aumento de 61,5\%. No estado de Alagoas, na série histórica de 1996 a 2016, foi registrada uma RMM de 47,63 óbitos/ 100 mil NV, com tendência de crescimento e distribuição heterogênea entre os municípios e regiões de saúde do estado (Azevedo et al., 2020; Duarte et al., 2020).

Taxas elevadas nessa região podem estar relacionadas as deficiências nos índices de escolaridade, condições socioeconômicas, moradia, saneamento e dificuldades de acesso e utilização dos serviços de saúde, que ainda são destaques no Nordeste, o que faz com que seja esperada uma RMM ainda mais elevada do que as encontradas nos estudos (Guerra et al., 2016; Araújo et al., 2017; Rodrigues et al., 2017).

Resultados elevados de RMM também foram encontrados em outras regiões do país. Na região sudeste, estado de Minas Gerais, o município de Montes Claros registrou taxas elevadas de RMM no período de 2009 a 2013, com destaque para o ano de 2012 em que foram registrados 138,76 óbitos/ 100 mil NV (Ruas et al., 2020). Na região Norte, uma pesquisa realizada no Pará evidenciou dados semelhantes aos encontrados em Aracaju, em que aquele estado cursou com uma RMM média de 61,2 óbitos por 100 mil NV de 2005 a 2014 (Santos et al., 2017).

A MM ainda permanece como um grande desafio e que demanda ações urgentes e efetivas para sua redução. A nível mundial destaca-se os Objetivos de Desenvolvimento Sustentável (ODS), que é uma estratégia que sucede os Objetivos de Desenvolvimento do Milênio (ODM), em que se prorrogam e reafirmam os compromissos globais assumidos anteriormente. Nessa estratégia a redução da MM foi pactuada para 70 mortes/ 100 mil NV até o ano de 2030 e o Brasil assume como meta a redução para 30 mortes/ 100 mil NV (Instituto de Pesquisa Econômica Aplicada, 2018; World Health Organization, 2019).

Nesse sentido, o Brasil implementou algumas estratégias como a Rede Cegonha e o projeto Ápice On para a qualificação e aperfeiçoamento da assistência materno-infantil no país (Portaria $n^{\circ}$ 1.459, de 24 de junho de 2011, 2011; Brasil et al., 2020). Outra estratégia adotada foi a implantação dos Comitês de Mortalidade Materna que objetivam a investigação dos óbitos maternos, bem como a análise e apontamento de medidas de prevenção e são uma forma de subsidiar essas políticas públicas implantadas (Mascarenhas et al., 2017). 
No Brasil, as características sociodemográficas refletem também fatores de risco para a MM (Duarte et al., 2020). Com relação ao perfil sócio demográfico, o estudo evidenciou uma média de idade de 29,47 ( \pm 7.19$)$ anos, variando entre 15 a 41 anos. Predominou mulheres de cor Preta/Parda ( $\mathrm{n}=39 ; 81,25 \%)$, solteiras $(\mathrm{n}=29 ; 59,18 \%)$, com nível de escolaridade de 8 a 11 anos de estudo ( $(n=20 ; 40,82 \%)$ e situação ocupacional "donas de casa" ( $n=23 ; 51,11 \%)$. Em um óbito não foi registrada a cor da mulher e em 04 óbitos não foi registrada a ocupação da mulher (Tabela 2).

Tabela 1: Caracterização sociodemográfica dos óbitos maternos em Aracaju de 2010 a 2018.

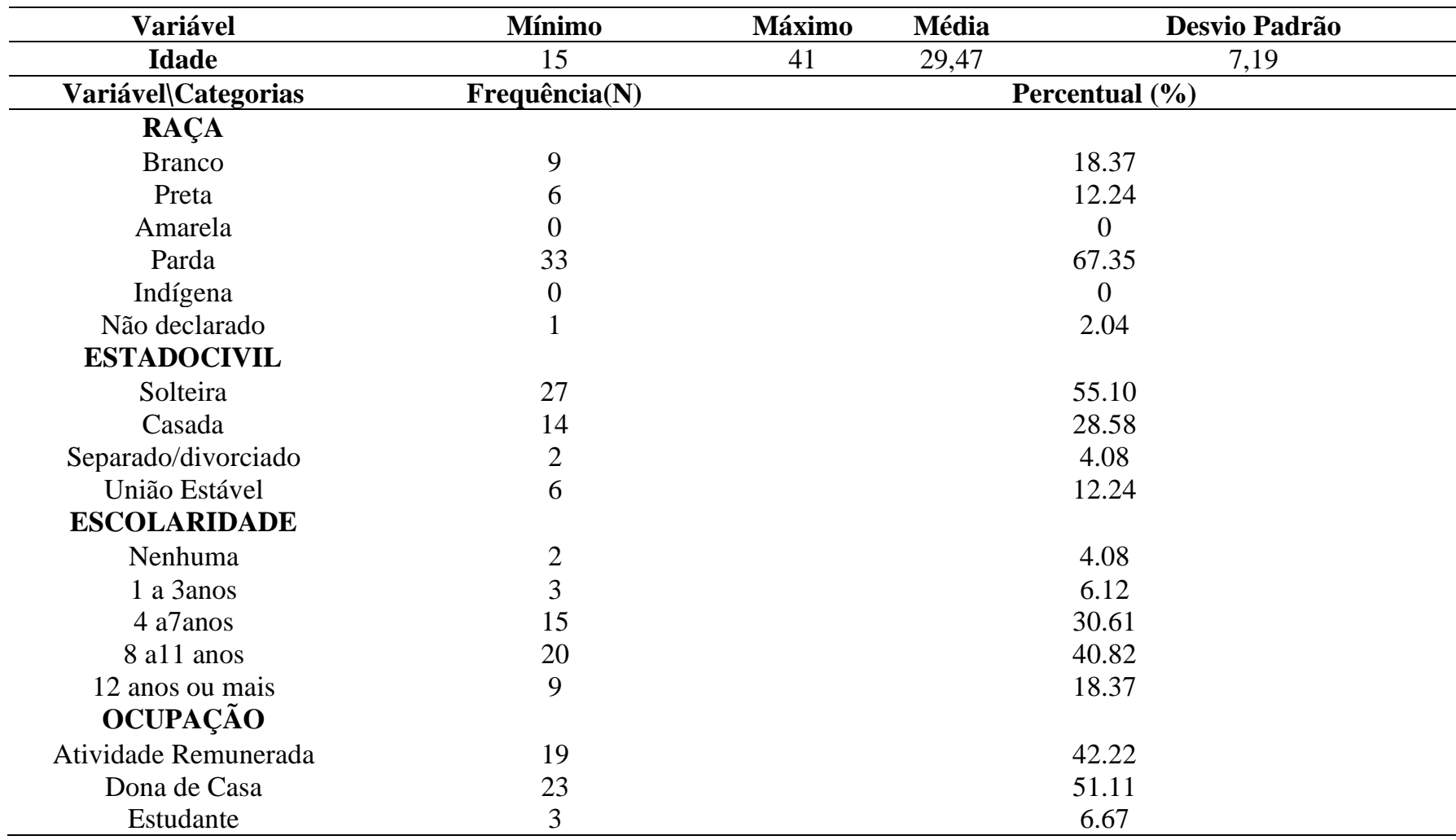

Fonte: Vigilância Epidemiológica municipal (2019).

A idade constitui um fator imprescindível na análise de óbitos maternos, sobretudo quando direcionado para mulheres que cursam com gestação nos extremos da vida reprodutiva, fato observado neste estudo. O desenvolvimento da gestação em mulheres com idade avançada é considerado de risco elevado, em virtude de alterações que proporcionam síndromes hipertensivas, ruptura de membranas, diabetes e chance elevada do índice de Apgar no quinto minuto ser menor que sete. $\mathrm{O}$ fator idade duplica o risco para mortalidade por problemas decorrentes da gravidez e parto (Gonçalves \& Monteiro, 2012; Gravena et al., 2013; Trigo et al., 2020).

A baixa escolaridade favorece uma gestação de alto risco e a MM, pois pode predispor a escassez do aprendizado, acesso a informações, prevenção de doenças e promoção da saúde, formulação de conceitos e princípios referentes ao autocuidado, aspectos que refletem diretamente na adesão pré-natal (Leite et al., 2011; Viana et al., 2011; Melo et al., 2016; Mascarenhas et al., 2017; de Oliveira et al., 2019).

No estudo foi identificado maior número de óbitos em mulheres que apresentaram escolaridade entre 8 a 11 anos de estudos, fato este diferente do encontrado a nível nacional, em que a MM ocorre mais em mulheres com escolaridade inferior a 7 anos de estudos (Carreno et al., 2014; Araújo et al., 2017; Mascarenhas et al., 2017; Scarton et al., 2019).

Pôde-se observar também o predomínio da população preta/parda com relação ao quantitativo de óbitos correspondendo a $81,25 \%$ do total. É sabido que a população negra ainda se encontra em um contexto de maior vulnerabilidade somado ao fato 
de comporem também uma parcela de mulheres que tem o acesso aos serviços de saúde dificultoso, que repercute negativamente no acesso aos método contraceptivos, fatos que proporcionam maior chance de gravidez, como identificado no estudo. As dificuldades que esse grupo racial enfrenta interfere no ciclo gravídico-puerperal, que consequentemente incorre em prejuízos na qualidade da atenção à saúde que é ofertada para essas mulheres (Batista et al., 2016; Leal et al., 2017; Mendes \& Eduardo, 2020; Nascimento, 2019).

A pesquisa Nascer no Brasil: Pesquisa Nacional sobre Parto e Nascimento, realizada em 2011/2012 com 23,894 mulheres revelou que, as mulheres de cor preta e parda apresentaram os piores indicadores de atenção pré-natal e parto em comparação às mulheres brancas. As puérperas de cor preta possuíram maior risco de pré-natal inadequado, falta de vinculação à maternidade e ausência de acompanhante. Foram identificadas desigualdades raciais no processo de atenção à gestação e ao parto evidenciando um gradiente de pior para melhor cuidado entre mulheres pretas, pardas e brancas (Leal et al., 2018).

Estudos realizados no Brasil revelam de igual modo esse destaque da população negra em relação a RMM. Um estudo realizado no Mato Grosso demonstrou que 63\% dos óbitos maternos ocorridos entre anos 2000 e 2006 deram-se entre as afrodescendentes. De igual modo, outro estudo realizado em Jequié na Bahia revelou destaque de RMM para a população preta/parda. Um estudo realizado nas cidades de Belo Horizonte e Uberaba evidenciou também uma maior incidência de óbitos em mulheres de raça cor parda e preta (Teixeira et al., 2012; Biano et al., 2017; Mascarenhas et al., 2017).

Quando considerado o momento de ocorrência do óbito, de acordo com a DO, a maioria ocorreu no Puerpério ( $\mathrm{n}=44$; $78,21 \%$ ), e todos ocorreram em estabelecimento hospitalar (Tabela 03). Tais fatos são semelhantes aos encontrados na cidade de Ribeirão Preto, São Paulo, no período 2000 a 2012 em que cerca de 81\% dos óbitos maternos ocorreram no puerpério. Um estudo realizado na Bahia evidenciou que $86,7 \%$ ocorreram em estabelecimento hospitalar. Destaca-se que o período do puerpério é crítico de risco para MM e ressalta-se a importância da continuidade do cuidado de forma qualificada no pós-parto (Coelho et al., 2016; Fernandes et al., 2015; Matoso, 2020).

Após a investigação do Comitê de Mortalidade Materna foi evidenciado que a maioria dos óbitos ocorreu no puerpério $(\mathrm{n}=40 ; 81,63 \%)$ em estabelecimento público $(\mathrm{n}=40 ; 81,63 \%)$. Quanto à distribuição da evitabilidade do óbito, observou-se que 67,35\% (n=33) seriam provavelmente evitáveis; 18,37\% $(\mathrm{n}=9)$ evitáveis e 12,23\% $(\mathrm{n}=6)$ provavelmente inevitáveis. Dentre os fatores de evitabilidade destacou-se o Institucional $(n=38 ; 21,72 \%)$. Em relação a classificação dos óbitos, $79,59 \%$ $(\mathrm{n}=39)$ foram classificados como Obstétrico Direto (Tabela 04).

Quanto às características obstétricas e dos serviços de saúde, $81,63 \%(\mathrm{n}=40)$ das mulheres realizaram pré-natal, destas 48,98\% ( $\mathrm{n}=24)$ tiveram assistência considerada inadequada neste período. Quanto a assistência o parto, 75,51\% $(\mathrm{n}=37)$ ocorreram em estabelecimentos públicos, sendo 48,86\% $(n=21)$ dos casos avaliados como assistência inadequada (Tabela 04).

Tabela 2: Caracterização óbitos materno em Aracaju de 2010 a 2018 segundo a declaração de óbito.

\begin{tabular}{lcc}
\hline Variável\Categorias & Frequência(N) & Percentual (\%) \\
\hline MOMENTO DO ÓBITO & 11 & 19,65 \\
Gravidez & 38 & 67,85 \\
Puerpério, até42diasapósoparto & 6 & 10,71 \\
Puerpério, de43diasa1ano & 1 & 1,79 \\
Ignorado & & 100 \\
LOCALDEOCORRÊNCIADO ÓBITO & 49 & \\
Hospital & 49
\end{tabular}


Tabela 3: Caracterização dos óbitos maternos em Aracaju de 2010 a 2018 segundo investigação do Comitê de mortalidade materna.

\begin{tabular}{|c|c|c|}
\hline Variável\Categorias & Frequência(N) & Percentual (\%) \\
\hline \multicolumn{3}{|l|}{ MOMENTO DO ÓBITO } \\
\hline Gravidez & 6 & 12.24 \\
\hline Puerpério & 40 & 81.63 \\
\hline Aborto & 2 & 4.08 \\
\hline Pós-Aborto & 1 & 2.04 \\
\hline \multicolumn{3}{|c|}{ ESTAB. DE OCORRÊNCIA DO PARTO/ABORTO } \\
\hline Público & 37 & 75.51 \\
\hline Privado & 10 & 20.41 \\
\hline Ignorado & 2 & 4.08 \\
\hline \multicolumn{3}{|l|}{ ESTAB. DE OCORRÊNCIA DO ÓBITO } \\
\hline Público & 40 & 81.63 \\
\hline Privado & 8 & 16.32 \\
\hline Ignorado & 1 & 2.05 \\
\hline \multicolumn{3}{|l|}{ PRÉ-NATAL } \\
\hline Não & 8 & 16.32 \\
\hline Sim, em estabelecimento público & 26 & 53.06 \\
\hline Sim, em estabelecimento privado & 9 & 18.36 \\
\hline Sim, em estabelecimento público e privado & 5 & 10.21 \\
\hline Ignorado & 1 & 2.05 \\
\hline \multicolumn{3}{|l|}{ PRÉ-NATAL ADEQUADO } \\
\hline Inconclusivo & 10 & 20.41 \\
\hline Não & 24 & 48.98 \\
\hline Sim & 10 & 20.41 \\
\hline Não se aplica & 3 & 6.12 \\
\hline Ignorado & 2 & 4.08 \\
\hline \multicolumn{3}{|l|}{ PARTO DE ASSISTÊNCIA ADEQUADA } \\
\hline Inconclusivo & 5 & 10.20 \\
\hline Não & 21 & 42.86 \\
\hline Sim & 19 & 38.77 \\
\hline Não se aplica & 1 & 2.05 \\
\hline Ignorado & 3 & 6.12 \\
\hline \multicolumn{3}{|l|}{ EVITABILIDADE } \\
\hline Provavelmente Evitável & 33 & 67.35 \\
\hline Evitável & 9 & 18.37 \\
\hline Provavelmente inevitável & 6 & 12.23 \\
\hline Inconclusivo & 1 & 2.05 \\
\hline \multicolumn{3}{|l|}{ FATORES DE EVITABILIDADE } \\
\hline Familiar & 27 & 15.43 \\
\hline Gestante & 26 & 14.85 \\
\hline Comunidade & 26 & 14.85 \\
\hline Social & 17 & 9.72 \\
\hline Institucional & 38 & 21.72 \\
\hline Profissional & 26 & 14.86 \\
\hline Intersetorial & 14 & 8.00 \\
\hline Inconclusivo & 1 & 0.57 \\
\hline \multicolumn{3}{|l|}{ CLASSIFICAÇÃO DO ÓBITO } \\
\hline Obstétrico Direto & 39 & 79.59 \\
\hline Obstétrico Indireto & 7 & 14.29 \\
\hline Materno descartado & 2 & 4.08 \\
\hline Tardio & 1 & 2.04 \\
\hline \multicolumn{3}{|l|}{ ÓBITO NA DECLARAÇÃO DE ÓBITO } \\
\hline Não & 12 & 24.49 \\
\hline Sim & 37 & 75.51 \\
\hline
\end{tabular}

Fonte: Vigilância Epidemiológica municipal (2019). 
Sabe-se que no Brasil, as causas obstétricas diretas merecem destaque por apresentar uma elevada incidência, girando em trono de 67\% (Ruas et al., 2020). Um estudo realizado em Campinas, de 2000 a 2015 revelou que as causas obstétricas diretas foram responsáveis por 54,2\% dos óbitos (I. M. G. D. de Oliveira, 2016). Já em Goiás, no período de 2010 a 2016, essas causas foram responsáveis por 55\% dos óbitos maternos. Estudos realizados em Ribeirão Preto/SP, Bahia e Amazonas evidenciaram também uma elevada incidência dessas causas, sendo $56,8 \%, 62,8 \%$ e $67,19 \%$ respectivamente, corroborando com os resultados encontrados em Aracaju/SE (Coelho et al., 2016; Fernandes et al., 2015; Medeiros et al., 2018).

Elevados índices podem representam uma falha dos serviços de saúde em relação às causas preveníveis e merecem destaque (Lima et al., 2017). É importante destacar que 67,35\% desses óbitos foram considerados provavelmente evitáveis, $18,37 \%$ como evitáveis e o principal fator de evitabilidade dos óbitos evidenciado no estudo foi o fator institucional, que diz respeito à infraestrutura do serviço e disponibilidade de recursos adequados para a assistência à mulher, como protocolos institucionais, leitos de UTI e educação permanente em saúde.

No que se refere às características obstétricas, notou-se que a maioria dos óbitos ocorreu no puerpério $(81,63 \%)$. Este fato evidencia a importância do acompanhamento pós-parto, pois nesse período ainda permanecem os fatores de risco que podem gerar complicações e ocasionar o óbito (Lima et al., 2017; Mascarenhas et al., 2017).

A grande maioria realizou o pré-natal, porém $48,98 \%$ foi considerado inadequado. A qualificação da assistência prestada às mulheres durante o ciclo gravídico puerperal é alvo primordial para redução da mortalidade materna, que deve incluir cobertura efetiva, ampla disponibilidade, custo acessível, qualidade e segurança (Dias et al., 2014)

O georreferenciamento tem sido utilizado para análise da distribuição espacial, permitindo a caracterização da condição de saúde em determinada região (Inagaki et al., 2014). A identificação dos bairros com maiores taxas de MM é uma ferramenta essencial para o desenvolvimento de políticas públicas e programas para a redução da MM. A visualização gráfica permite a identificação de desigualdades intraurbanas e quando associadas a seus fatores possibilita a orientação das políticas públicas para intervenções específicas (Borges, 2012).

Figura 1: Distribuição espacial dos óbitos maternos em Aracaju de 2010 a 2018.

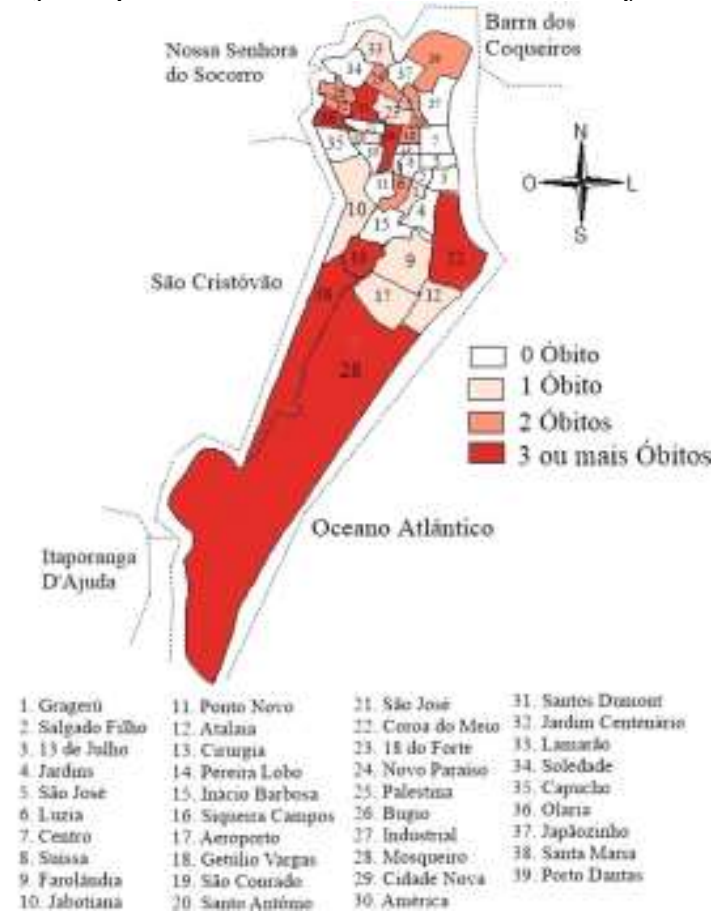

Fonte: Autores. 
Figura 2: Densidade de Kernel dos óbitos maternos em Aracaju de 2010 a 2018.

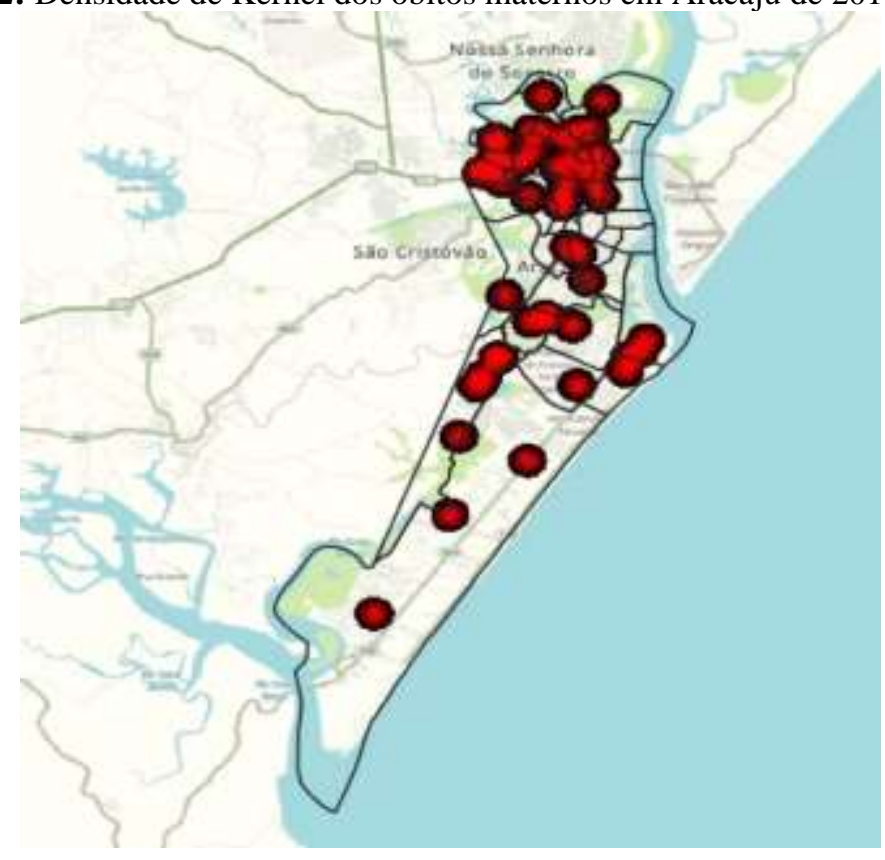

Fonte: Autores.

O estudo evidenciou que a MM apresentou uma distribuição variável entre os bairros do município (Figura 1). Esse quadro sugere relação entre baixa condição socioeconômica da população e MM. Essas informações são importantes por evidenciar as áreas do município mais vulneráveis e que mais demandam ações voltadas para a redução da MM.

Houve concentração dos mais elevados índices de MM em bairros considerados de baixa e muito baixa condição de vida como Santa Maria, Santos Dumont e São Conrado, que concentraram 3 ou mais óbitos no período analisado, e por sua vez são bairros com menor poder socioeconômico (Aracaju, 2018). Nos bairros considerados nobres como Jardins, Pereira Lobo e 13 de Julho não foi registrado nenhum óbito. Porém, mesmo em bairros considerados de melhores condições socioeconômicas como Luzia e Atalaia foram registrados 2 e 1 óbitos respectivamente (Aracaju, 2018).

Com relação à densidade de Kernel (Figura 2), foi possível observar que as regiões Norte e Oeste do município apresentaram a maior densidade de óbitos. Isso pode estar relacionado ao maior número de bairros encontrados na região em contraste a região Sul e Leste.

Algumas limitações enfrentadas no desenvolvimento do estudo estão relacionadas a subnotificação e subregistro encontrados nos dados referentes ao óbito materno. Somado a isso, a indisponibilidade de dados mais atualizados referentes ao Censo Demográfico sobre estratos e condições de vida dos bairros dificultaram a análise referente a distribuição espacial dos óbitos.

Salienta-se que registros incompletos nos Sistemas de Informação em Saúde (SIS) são preocupantes, tendo em vista que nos últimos 10 anos houve um aumento de análise dos SIS considerando a importância dessas estatísticas e da análise desses dados. A subnotificação de óbitos e subdeclarações das causas maternas são pontos que acabam subestimando a MM e constituem-se também como um desafio para a redução desse agravo, pois é necessário conhecer os reais dados e a magnitude da problemática para que as políticas públicas e estratégias construídas sejam eficazes (Matoso, 2020).

Como contribuições o estudo permite subsidiar ações e programas mais específicos para a redução da MM no município, por apresentar o perfil epidemiológico da mortalidade, possíveis fatores de risco e associações, bem como a distribuição espacial e áreas de maior acometimento. 


\section{Conclusão}

A MM é vista como uma grave violação dos direitos humanos justamente por ser considerada uma fatalidade evitável. O presente estudo permitiu verificar que a MM no município de Aracaju, mesmo diante da redução no ano de 2012, manteve-se acima da média preconizada pelos ODS, fato que merece relevância. O destaque para as causas obstétricas diretas evidencia o déficit na assistência à saúde materna no município. O predomínio da população de raça cor preta/parda chama a atenção para a vulnerabilidade desse grupo, a necessidade de melhor qualificação do acompanhamento dessas mulheres no ciclo gravídicopuerperal no município e de melhoria do acesso dessas mulheres ao serviço de saúde. O perfil dos óbitos maternos ressalta a importância de um maior empenho das políticas públicas, profissionais de saúde e sociedade como um todo, objetivando reduzir esses índices não só no município de Aracaju, mas também a nível nacional.

\section{Referências}

Aracaju. (2018). Índice Cadúnicode Condições de Vida. https://www.aracaju.se.gov.br/userfiles/observatorio/arquivos/OBSERVATORIO-Relatorio-CadonicoA4.pdf

Araújo, A. J. de S., Sena, Á. A., Santana, I. T., \& Barreto, É. de S. (2017). The magnitude of maternal mortality in Bahia in the last 10 years. RevEnferm UFPI, 6(2), Article 2. https://periodicos.ufpi.br/index.php/reufpi/article/view/439

Azevedo, L. M. C., Souza, D. R. X. de, Bezerra, K. K. S., Batista, H. M. T., Sousa, E. S. S., Bezerra, A. M. F., \& Leite, E. de S. (2020). Distribuição da Mortalidade Materna no Estado da Paraíba no período de 2007 a 2016 / Distribution of Maternal Mortality in Paraiba State from 2007 to 2016. Revista de psicologia, 14(51), 486-501. https://doi.org/10.14295/idonline.v14i51.2614

Barreto, B. L. (2021). Perfil epidemiológico da mortalidade materna no Brasil no período de 2015 a 2019. Revista Enfermagem Contemporânea, 10(1), 127133. https://doi.org/10.17267/2317-3378rec.v10i1.3709

Batista, L. E., Rattner, D., Kalckmann, S., \& Oliveira, M. C. G. de. (2016). Humanização na atenção à saúde e as desigualdades raciais: Uma proposta de intervenção. Saúde e Sociedade, 25, 689-702. https://doi.org/10.1590/s0104-12902016146290

Biano, R. K. C., Souza, P. C. B. de, Ferreira, M. B. G., Silva, S. R. da, \& Ruiz, M. T. (2017). Mortalidade materna no Brasil e nos municípios de Belo Horizonte e Uberaba, 1996 a 2012. Revista de Enfermagem do Centro-Oeste Mineiro, 7(0), Article 0. https://doi.org/10.19175/recom.v7i0.1464

Borges, C. L. M. S. (2012). Epidemiologia e gestão: Análise da mortalidade materna em Aracaju [Dissertação de Mestrado, Universidade Federal da Bahia]. http://repositorio.ufba.br/ri/handle/ri/6851

Brasil (Org.). (2011). Política nacional de atenção integral à saúde da mulher: Princípios e diretrizes. Editora MS. http://bvsms.saude.gov.br/bvs/publicacoes/politica_nacional_mulher_principios_diretrizes.pdf

Brasil, \& Ministério da Saúde. (2009). Manual dos comitês de mortalidade materna. Ministério da Saúde. http://bvsms.saude.gov.br/bvs/publicacoes/guia_vigilancia_epidem_obito_materno.pdf

Brasil, Ministério da Saúde, \& Secretaria de Vigilância em Saúde. (2020). Boletim epidemiolígico N 20 (Vol. 51). Ministério da Saúde.

Brasil. (2007). Objetivos de desenvolvimento do milenio - Principais iniciativas do governo federal. http://www.abc.gov.br/api/publicacaoarquivo/7

Brasil. (2019). Departamento de Informática do Sistema Único de Saúde. http://www2.datasus.gov.br/DATASUS/index.php?area0205

Carreno, I., Bonilha, A. L. de L., \& Costa, J. S. D. da. (2014). Evolução temporal e distribuição espacial da morte materna. Revista de Saúde Pública, 48, 662670. https://doi.org/10.1590/S0034-8910.2014048005220

Coelho, V. C., Andrade, M. S., Sena, C. D. de, Costa, L. E. L., \& Bittencourt, I. S. (2016). Caracterização Dos Óbitos Maternos Em Três Regiões De Saúde Do Centro-Norte Baiano. Cogitare Enfermagem, 21(1), Article 1. https://doi.org/10.5380/ce.v21i1.42475

Dias, J. M. G., Oliveira, A. P. S. de, Cipolotti, R., Monteiro, B. K. S. M., \& Pereira, R. de O. (2014). Mortalidade materna. 25(2), 173-179. https://doi.org/10.5935/2238-3182.20150034

Duarte, E. M. da S., Alencar, É. T. dos S., Fonseca, L. G. A. da, Silva, S. M. da, Machado, M. F., Araújo, M. D. P. de, Correia, D. S., \& Souza, C. D. F. de. (2020). Mortalidade materna e vulnerabilidade social no Estado de Alagoas no Nordeste brasileiro: Uma abordagem espaço-temporal. Revista Brasileira de Saúde Materno Infantil, 20, 575-586. https://doi.org/10.1590/1806-93042020000200014

Fernandes, B. B., Nunes, F. B. B. de F., Prudêncio, P. S., \& Mamede, F. V. (2015). Pesquisa epidemiológica dos óbitos maternos e o cumprimento do quinto objetivo de desenvolvimento do milênio. Revista Gaúcha de Enfermagem, 36, 192-199. https://doi.org/10.1590/1983-1447.2015.esp.56792

Freitas-Júnior, R. A. de O. (2020). Mortalidade materna evitável enquanto injustiça social. Revista Brasileira de Saúde Materno Infantil, 20 , 607-614. https://doi.org/10.1590/1806-93042020000200016

Gonçalves, Z. R., \& Monteiro, D. L. M. (2012). Complicações maternas em gestantes com idade avançada. Femina. http://files.bvs.br/upload/S/01007254/2012/v40n5/a3418.pdf 
Gravena, A. A. F., Paula, M. G. de, Marcon, S. S., Carvalho, M. D. B. de, \&Pelloso, S. M. (2013). Idade materna e fatores associados a resultados perinatais. Acta Paulista de Enfermagem, 26, 130-135. https://doi.org/10.1590/S0103-21002013000200005

Guerra, H. S., Hirayama, A. B., Silva, A. K. C. da, Oliveira, B. de J. S., \& Oliveira, J. F. de J. (2016). Análise das Ações da Rede Cegonha no Cenário Brasileiro. Iniciação Cientifica Cesumar, 18(1), 73-80. https://doi.org/10.17765/1518-1243.2016v18n1p73-80

Inagaki, A. D. de M., Cardoso, N. P., Lopes, R. J. P. L., Alves, J. A. B., Mesquita, J. R. F., Araújo, K. C. G. M. de, \&Katagiri, S. (2014). Análise espacial da prevalência de toxoplasmose em gestantes de Aracaju, Sergipe, Brasil. Revista Brasileira de Ginecologia e Obstetrícia, 36, 535-540. https://doi.org/10.1590/So100-720320140005086

Instituto Brasileiro de Geografia e Estatística. (2010). Censo demográfico. https://cidades.ibge.gov.br/brasil/se/aracaju/panorama

Instituto de Pesquisa Econômica Aplicada. (2018). Agenda 2030 - ODS-Metas Nacionais dos Objetivos de Desenvolvimento Sustentável. Ministério da Saúde. https://www.ipea.gov.br/portal/images/stories/PDFs/livros/livros/180801_ods_metas_nac_dos_obj_de_desenv_susten_propos_de_adequa.pdf

Leal, M. do C., Gama, S. G. N. da, Pereira, A. P. E., Pacheco, V. E., Carmo, C. N. do, \& Santos, R. V. (2017). A cor da dor: Iniquidades raciais na atenção prénatal e ao parto no Brasil. Cadernos de Saúde Pública, 33. https://doi.org/10.1590/0102-311X00078816

Leal, M. do C., Szwarcwald, C. L., Almeida, P. V. B., Aquino, E. M. L., Barreto, M. L., Barros, F., \&Victora, C. (2018). Saúde reprodutiva, materna, neonatal e infantil nos 30 anos do Sistema Único de Saúde (SUS). Ciência \& Saúde Coletiva, 23, 1915-1928. https://doi.org/10.1590/1413-81232018236.03942018

Leite, R. M. B., Araújo, T. V. B. de, Albuquerque, R. M. de, Andrade, A. R. S. de, \& Duarte Neto, P. J. (2011). Fatores de risco para mortalidade materna em área urbana do Nordeste do Brasil. Cadernos de Saúde Pública, 27, 1977-1985. https://doi.org/10.1590/S0102-311X2011001000011

Lima, M. R. G. de, Coelho, A. S. F., Salge, A. K. M., Guimarães, J. V., Costa, P. S., Sousa, T. C. C. de, Mattos, D. V. de, \& Sousa, M. A. A. (2017). Alterações maternas e desfecho gravídico-puerperal na ocorrência de óbito materno. Cadernos Saúde Coletiva, 25, 324-331. https://doi.org/10.1590/1414$462 \times 201700030057$

Mann, H. B., \& Whitney, D. R. (1947). On a Test of Whether one of Two Random Variables is Stochastically Larger than the Other. The Annals of Mathematical Statistics, 18(1), 50-60.

Mascarenhas, P. M., Silva, G. R. da, Reis, T. T., Casotti, C. A., \& Nery, A. A. (2017). Análise da mortalidade materna. Rev. enferm. UFPE online, $4653-4662$.

Matoso, L. (2020). Mortalidade Materna No Estado Do Rio Grande Do Norte: Um Estudo Retrospectivo (2015-2018). Revista Ciência e Desenvolvimento, 13, 476-495. https://doi.org/10.11602/1984-4271.2020.13.2.13

Medeiros, L. T., Sousa, A. M., Arinana, L. O., Inácio, A. S., Prata, M. do L. C., \& Vasconcelos, M. N. G. (2018). Mortalidade Materna No Estado Do Amazonas: Estudo Epidemiológico. Revista Baiana de Enfermagem32, . https://doi.org/10.18471/rbe.v32.26623

Melo, W. A. de, Alves, J. I., Ferreira, A. A. da S., Souza, V. S. de, \&Maran, E. (2016). Gestação de alto risco: Fatores associados em município do noroeste paranaense. Espaço para Saúde, 17(1), 83-92. https://doi.org/10.22421/15177130-2016v17n1p83

Mendes, A. G. de S., \& Eduardo, G. N. (2020). Vidas Negras Importam! Um estudo sobre os indicadores sociais da mortalidade materna de mulheres negras na Paraíba. Cadernos de Gênero e Diversidade, 6(1), 69-83. https://doi.org/10.9771/cgd.v6i1.27817

Nascimento, M. M. do. (2019). Mortalidade Materna Da Mulher Negra Em Japeri [Dissertação (Mestrado), Centro Federal de Educação Tecnológica Celso Suckow da Fonseca]. http://dippg.cefet-rj.br/pprer/attachments/article/81/139_Mariane\%20Mara\%CC\%81al\%20do\%20Nascimento.pdf

Oliveira, A. S. de, Lima, D. J. J., \& Menezes, N. C. (2019). Perfil epidemiológico dos casos de morte materna na cidade de Manaus por: Causa, escolaridade e raça, no período de 2011 a 2015. Revista Eletrônica Acervo Saúde, 23, e424. https://doi.org/10.25248/reas.e424.2019

Oliveira, I. M. G. D. de. (2016). Fatores associados à mortalidade materna em Campinas/SP entre 2000 e 2015 [Dissertação de Mestrado, UNICAMP]. http://repositorio.unicamp.br/jspui/handle/REPOSIP/325026

Pearson, K. (1900). X. On the criterion that a given system of deviations from the probable in the case of a correlated system of variables is such that it can be reasonably supposed to have arisen from random sampling. The London, Edinburgh, and Dublin Philosophical Magazine and Journal of Science, 50(302), 157175. https://doi.org/10.1080/14786440009463897

Portaria N 1.119, de 5 de junho de 2008, Regulamenta a vigilância de óbitos maternos (2008) (testimonyof Brasil \& Ministério da Saúde).

Portaria $n^{o}$ 1.459, de 24 de junho de 2011, Institui, no âmbito do Sistema Único de Saúde - SUS - a Rede Cegonha (2011) (testimonyof Brasil \& Ministério da Saúde).

QGIS Development Team. (2019). QGIS User Guide, Release 3.8: QGIS Project.

Rodrigues, A. R. M., Cavalcante, A. E. S., \& Barbosa, A. (2019). Mortalidade materna no Brasil entre 2006-2017: Análise temporal. ReTEP, 11(1), 3-9.

Rodrigues, A. R. M., Dantas, S. L. da C., Pereira, A. M. M., Silveira, M. A. M. da, \& Rodrigues, D. P. (2017). High-Risk Pregnancy: Analysis Of Health Determinants. 16(1), 6.

Ruas, C. A. M., Quadros, J. F. C., Rocha, J. F. D., Rocha, F. C., Andrade Neto, G. R. de, Piris, Á. P., Rios, B. R. M., Pereira, S. G. S., Ribeiro, C. D. A. L., \& Leão, G. M. M. S. (2020). Profile andspatialdistributionon maternal mortality. Revista Brasileira de Saúde Materno Infantil, 20, 385-396. https://doi.org/10.1590/1806-93042020000200004

Santos, D. R. dos, Nogueira, L. M. V., Paiva, B. L., Rodrigues, I. L. A., Oliveira, L. F. de, \& Caldas, S. P. (2017). Maternal mortality in the indigenous and nonindigenous population in Pará: Contribution to the surveillance of deaths. Escola Anna Nery, 21(4). https://doi.org/10.1590/2177-9465-ean-2017-0161 
Research, Society and Development, v. 10, n. 12, e373101220657, 2021

(CC BY 4.0) | ISSN 2525-3409 | DOI: http://dx.doi.org/10.33448/rsd-v10i12.20657

Scarton, J., Paula, S. F. de, Andrade, G. B. de, Rangel, R. F., Ventura, J., \& Siqueira, H. C. H. de. (2019). Maternal Mortality Profile: AnIntegrativeLiterature Review / Perfil da Mortalidade Materna: Uma Revisão Integrativa da Literatura. Revista de Pesquisa Cuidado é Fundamental Online, 11(3), 816-822. https://doi.org/10.9789/2175-5361.2019.v11i3.816-822

Teixeira, N. Z. F., Pereira, W. R., Barbosa, D. A., \& Vianna, L. A. C. (2012). Mortalidade materna e sua interface com a raça em Mato Grosso. Revista Brasileira de Saúde Materno Infantil, 12(1), 27-35. https://doi.org/10.1590/S1519-38292012000100003

The R core team. (2018). R: A Language and Environment for Statistical Computing.

Torres, N. M. F., Santos, J. L. da C. dos, Silva, B. L., Silva, P. T. da, Linhares, B. O., Silva, A. L. F. da, \& Moura, M. C. L. de. (2021). Mortalidade materna no Nordeste brasileiro. Revista de Casos e Consultoria, 12(1), e23821-e23821.

Trigo, I. G., Eller, J. X., Vaz, M. R., Calil, C., Silva, L. R., \& Barboza, B. de P. (2020). Idade Materna Avançada E Seus Desfechos. Cadernos da Medicina UNIFESO, 2(3), Article 3. http://www.revista.unifeso.edu.br/index.php/cadernosdemedicinaunifeso/article/view/1691

Vanderlei, L. C. de M., \& Frias, P. G. (2015). Avanços e desafios na saúde materna e infantil no Brasil. Revista Brasileira de Saúde Materno Infantil, 15, 157158. https://doi.org/10.1590/S1519-38292015000200001

Viana, R. da C., Novaes, M. R. C. G., \&Calderon, I. de M. P. [UNESP. (2011). Mortalidade Materna: Uma abordagem atualizada. Comunicação em Ciências da Saúde, 141.

World Health Organization. (2019). Trends in maternal mortality: 2000 to 2017. WHO. https://www.unfpa.org/sites/default/files/pubpdf/Maternal_mortality_report.pdf. 